\title{
Critical Point Approximation Through Exact Regularization
}

\author{
By Enrique Fernandez-Cara and Carlos Moreno
}

\begin{abstract}
We present several iterative methods for finding the critical points and/or the minima of a functional which is essentially the difference between two convex functions. The underlying idea relies upon partial and exact regularization of the functional, which allows us to preserve the local feature in a large number of applications, as well as to obtain some convergence results. These methods are further applied to some differential problems of the semilinear elliptic type arising in plasma physics and fluid mechanics.
\end{abstract}

1. Introduction. The main objective in this paper is to construct iterative algorithms for solving nondifferentiable extremal problems. From a theoretical point of view it is always possible to reformulate any such problem as an equivalent differentiable convex problem by means of the use of infimal convolution.

More precisely, with $H$ being a real Hilbert space and $J: H \rightarrow(-\infty, \infty]$ a proper lower semicontinuous (lsc) convex function, one has

$$
\inf _{l, H} J(v)=\inf _{v, w \in H}\left\{J(v)+\frac{1}{2 \lambda}|v-w|_{H}^{2}\right\} \equiv \inf _{w \in H} J_{\lambda}(w)
$$

for all $\lambda>0$. It is well known that $J_{\lambda}$ is $F$-differentiable and convex and $J_{\lambda}^{\prime}$ is Lipschitz-continuous with constant $1 / \lambda$. Furthermore, if $J$ is bounded from below, then

$$
\operatorname{Arg} \min _{w \in H} J_{\lambda}(w)=\operatorname{Arg} \min _{v \in H} J(v) .
$$

This argument allows us to use the (classical) methods in differentiable optimization to minimize $J$ over $H$. Thus, for the steepest descent algorithm, one is led to the following scheme:

$$
\begin{gathered}
u_{k+1}=u_{k}-\rho_{k} J_{\lambda}^{\prime}\left(u_{k}\right), \\
\rho_{k}=\operatorname{Arg} \min _{\rho \geqslant 0} J_{\lambda}\left(u_{k}-\rho J_{\lambda}^{\prime}\left(u_{k}\right)\right) .
\end{gathered}
$$

The fact that $J^{\prime}$ is $(1 / \lambda)$-Lipschitz-continuous has an important consequence on the size of $\rho_{k}$. Indeed, one must have $\rho_{k} \geqslant \lambda$. Therefore, it is reasonable to take $\rho_{k}=\lambda$ for all $k$, which leads to the proximal point algorithm:

$$
u_{k+1}=R_{\lambda}^{J}\left(u_{k}\right) \equiv u_{k}-\lambda J_{\lambda}^{\prime}\left(u_{k}\right) \text {. }
$$

Received July 17, 1984; revised February 25, 1987.

1980) Mathe'matics Subject (lassification (1985 Rerision). Primary 65N30).

Kel words and phrases. Nonconvex optimization, exact regularization, semilinear elliptic problems with discontinuous nonlinearities, plasma confinement, vortex rings.

(1) 1988 American Mathematical Socicty $0025-5718 / 88 \$ 1.00+\$ .25$ per page 
The operator $R_{\lambda}^{J}$ is the proximal mapping or resolvent associated with the subdifferential of $J$, i.e., with the set

$$
\partial J(v)=\left\{q \in H ; J(w)-J(v) \geqslant(q, w-v)_{H} \forall w \in H\right\} .
$$

This algorithm has first been used by B. Martinet (see [26]; see also [31], [32]). Although it is not obvious that (1.3) provides a useful method for minimizing an arbitrary convex function, this scheme has an important particularization: the multipliers method of Hestenes and Powell (see [32]). Applications of (1.3) and some of its variants to the solution of elliptic variational inequalities can be found in [9], [13], [14], [18], [19], [21], [22], [25] among others.

Our goal in this paper is to show that these techniques can be carried over to some nonconvex problems. First we recall some fundamental facts about the convex case. In general, for a function $\phi: H \rightarrow(-\infty, \infty], \phi^{*}(q)=\sup _{v \in H}\left\{(q, v)_{H}-\phi(v)\right\}$ is the convex conjugate of $\phi$.

Let $f$ and $g$ be two proper lsc convex functions on $H$. We consider the (primal) problem

$$
\begin{aligned}
& \text { Minimize } J(v)=f(v)+g(v) \\
& \text { subject to } v \in H .
\end{aligned}
$$

Following Fenchel and Rockafellar (see [11], [33], [34]), one can associate with (1.4) the dual problem

$$
\begin{aligned}
& \text { Minimize } J^{*}(q)=g^{*}(q)+f^{*}(-q) \\
& \text { subject to } q \in H .
\end{aligned}
$$

One knows that (under certain reasonable conditions) for a solution $u$ (resp. $p$ ) of (1.4) (resp. (1.5)) the following extremality condition is satisfied:

$$
u \in \partial f^{*}(-p), \quad p \in \partial g(u) \text {. }
$$

The proximal point algorithm applied to (1.5) yields the Hestenes and Powell iteration:

$$
\begin{gathered}
\partial f\left(u_{k+1}\right)+p_{k+1} \ni 0, \\
p_{k+1}=g_{\lambda}^{\prime}\left(u_{k+1}+\lambda p_{k}\right) .
\end{gathered}
$$

We note that for quadratic, strongly convex $f$ (also under weaker conditions) one obtains convergence results for the following semiexplicit version of (1.6)-(1.7):

$$
\begin{gathered}
f^{\prime}\left(u_{k+1}\right)+p_{k}=0, \\
p_{k+1}=g_{\lambda}^{\prime}\left(u_{k+1}+\lambda p_{k}\right)
\end{gathered}
$$

(see [9]). It can be shown that the sequence $J^{*}\left(p_{k}\right)$ generated by (1.8)-(1.9) is monotone decreasing for $\lambda$ large enough.

We turn now to the nonconvex case. A great number of nondifferentiable extremal problems can be formulated as:

$$
\begin{aligned}
& \text { Minimize } J(v)=f(v)-g(v) \\
& \text { subject to } v \in H,
\end{aligned}
$$

where $f$ and $g$ are as above. Again, in this case, a dual problem can be introduced (see [41], [42]; see also [4]):

$$
\begin{aligned}
& \text { Minimize } J^{*}(q)=g^{*}(q)-f^{*}(q) \\
& \text { subject to } q \in H,
\end{aligned}
$$


and the extremality relations become

$$
u \in \partial f^{*}(p), \quad p \in \partial g(u) .
$$

In general, it is not easy to apply an implicit scheme for solving (1.10) or (1.11), because at each step we would have to solve a nonconvex problem. This difficulty disappears for the semiexplicit scheme:

$$
\begin{gathered}
\partial f\left(u_{k+1}\right) \ni p_{k}, \\
p_{k+1}=g_{\lambda}^{\prime}\left(u_{k+1}+\lambda p_{k}\right) .
\end{gathered}
$$

Now, the monotonicity of $J^{*}\left(p_{k}\right)$ is obtained without additional conditions on $\lambda$, since one has

$$
g^{*}\left(p_{k+1}\right)-f^{*}\left(p_{k+1}\right)+\frac{\lambda}{2}\left|p_{k}-p_{k+1}\right|_{H}^{2} \leqslant g^{*}\left(p_{k}\right)-f^{*}\left(p_{k}\right) .
$$

The fact that a positive parameter $\lambda$ is used in (1.13) may certainly decrease the speed of convergence with respect to a fixed-point scheme. Indeed, there is no reason to choose $\lambda>0$, except that it is in general necessary to make the definition of $g_{\lambda}^{\prime}$ meaningful. However, for some particular cases (see Section 3), this limitation can be removed.

Another class of extremal problems considered in this paper is the following:

$$
\begin{aligned}
& \text { Minimize } J(v)=f(v)-g(v) \\
& \text { subject to } v \in \partial K
\end{aligned}
$$

Here, $K \subset H$ is a closed convex set with nonempty interior and $\partial K$ is its boundary. According to J. B. Hiriart-Urruty [23], an optimality condition for any solution $u$ of $(1.15)$ is

$$
\partial f(u)-\partial g(u)+N(K ; u) \cap N\left(K^{c} ; u\right) \ni 0,
$$

where

$N(K ; u)$ is the normal cone to $K$ at $u$, i.e., the subdifferential of the indicator functional of $K$ at $u\left(N(K ; u)=\partial I_{K}(u)\right.$ with $I_{K}(v)=0$ if $v \in K, I_{K}(v)=\infty$ otherwise),

$N\left(K^{c} ; u\right)$ is the (generalized) normal cone to the closure of $K^{c}$ at $u$, i.e., the generalized gradient (in the sense of $\mathrm{F}$. $\mathrm{H}$. Clarke [10]) of the function "distance from $K^{c}$ " at $u$.

Under the following regularity assumption on $\partial K$ :

$$
N(K ; u) \cap N\left(K^{c} ; u\right) \text { is a linear subspace of dimension one, }
$$
relation (1.16) becomes

$$
\partial f(u)-\partial g(u)+\beta \eta(u) \ni 0,
$$

for some real constant $\beta$ and with $\eta(u)$ being (by definition) a normal vector to $\partial K$ at $u$. Using (1.18), one can formulate some iterative methods for (1.15) similar to (1.12)-(1.13) (for details, see Section 4).

The remainder of this paper is organized as follows. In Section 2 we present the general formulation and the convergence analysis of an algorithm of the kind (1.12)-(1.13). Next (see Section 3), we apply these results to the solution of some elliptic problems with discontinuous nonlinearities. In Section 4 we consider some 
constrained problems for which we again derive a "smoothing" iterative procedure. Finally, Sections 5 and 6 deal with the applications of these techniques to the numerical solution of two free boundary problems, originating respectively in plasma physics and fluid mechanics.

2. The Unconstrained Problem. Throughout the sequel, $V$ is a real reflexive Banach space, $V^{\prime}$ is its dual space and $B$ is a bounded linear operator from $V$ into $H$, a Hilbert space. The functions $f: V \rightarrow(-\infty, \infty]$ and $g: H \rightarrow(-\infty, \infty]$ will be assumed to be convex, proper and lsc, and we identify $H$ with its dual space. We set

$$
J(v)=f(v)-g(B v) \quad \forall v \in V .
$$

Whether or not the algorithms described in Section 1 are computationally feasible depends upon the availability of an efficient procedure for evaluating $g_{\lambda}^{\prime}$. This motivates the presence of $B$ in (2.1).

Our purpose is to find $u \in V$ satisfying

$$
\partial f(u)-\partial(g \circ B)(u) \ni 0
$$

(this is an inclusion in $V^{\prime}$ ). In particular, every point at which $J$ reaches its minimum (if it exists) satisfies (2.2). In accordance with the rules of the subdifferential calculus, one has

$$
\partial(g \circ B)(v) \supset B^{*} \partial g(B v) \quad \forall v \in V,
$$

where $B^{*}$ is the adjoint of $B$. Thus, it suffices to solve

$$
\partial f(u)-B^{*} \partial g(B U) \ni 0, \quad u \in V .
$$

We consider the following algorithm:

Algorithm 1 ( $\lambda$ is a fixed positive parameter).

(a) Choose $p_{0} \in H$.

(b) For given $k \geqslant 0$ and $p_{k} \in H$, compute $u_{k+1}$ and $p_{k+1}$ by

$$
\partial f\left(u_{k+1}\right) \ni B^{*} p_{k}, \quad p_{k+1}=g_{\lambda}^{\prime}\left(B u_{k+1}+\lambda p_{k}\right) .
$$

As in Section 1, $g_{\lambda}^{\prime}$ in (2.4) is the Yosida approximation of the maximal monotone operator $\partial g$, i.e.,

$$
g_{\lambda}^{\prime}=\frac{1}{\lambda}\left(\mathrm{Id}-R_{\lambda}^{g}\right), \quad R_{\lambda}^{g}=(\mathrm{Id}+\lambda g)^{-1} .
$$

Actually, to make (2.4) meaningful, an assumption is necessary asserting that $\partial f^{*}\left(B^{*} p_{k}\right) \neq \varnothing$. So, the following condition on $f$ and $B$ will be imposed:

$$
\text { Dom } \partial f^{*} \supset R\left(B^{*}\right) \text {. }
$$

ThEOREM 2.7. Assume that, together with (2.6), one has

$$
\begin{gathered}
\inf _{i, V} J(v) \geqslant-C>-\infty, \\
J \text { is coercive, i.e., } J(v) \rightarrow \infty \text { as } v \in V,|v|_{V} \rightarrow \infty, \\
B \in \mathscr{L}(V ; H) \text { is compact. }
\end{gathered}
$$

Then every sequence $\left\{u_{k}\right\}$ generated by Algorithm 1 possesses subsequences weakly convergent in $V$ whose limit points are critical in the sense of (2.3). 
Proof. Clearly, (2.4) is equivalent to

$$
\begin{gathered}
f\left(u_{k+1}\right)+f^{*}\left(B^{*} p_{k}\right)=\left(p_{k}, B u_{k+1}\right)_{H}, \\
p_{k+1} \in \partial g\left(B u_{k+1}+\lambda\left(p_{k}-p_{k+1}\right)\right) .
\end{gathered}
$$

The last formula can also be written as

$$
p_{k+1}=\operatorname{Arg} \min _{q \in H}\left\{g^{*}(q)+\frac{\lambda}{2}\left|q-p_{k}\right|_{H}^{2}-\left(q, B u_{k+1}\right)_{H}\right\} .
$$

Then, for $J^{*}=g^{*}-f^{*} \circ B^{*}$, one obtains the following chain of inequalities:

$$
\begin{aligned}
J^{*}\left(p_{k+1}\right) & \leqslant f\left(u_{k+1}\right)+g^{*}\left(p_{k+1}\right)-\left(p_{k+1}, B u_{k+1}\right)_{H} \\
& =g^{*}\left(p_{k+1}\right)-f^{*}\left(B^{*} p_{k}\right)+\left(p_{k}-p_{k+1}, B u_{k+1}\right)_{H} \\
& \leqslant J^{*}\left(p_{k}\right)-\frac{\lambda}{2}\left|p_{k}-p_{k+1}\right|_{H}^{2} .
\end{aligned}
$$

On the other hand,

$$
f\left(u_{k}\right)+g^{*}\left(p_{k}\right)-\left(p_{k}, B u_{k}\right) \geqslant f\left(u_{k}\right)-g\left(B u_{k}\right)=J\left(u_{k}\right) .
$$

Hence, $\left\{J^{*}\left(p_{k}\right)\right\}$ is decreasing and, by (2.8), bounded from below:

$$
J^{*}\left(p_{k}\right) \geqslant J\left(u_{k+1}\right) \geqslant-C .
$$

Consequently, $J^{*}\left(p_{k}\right)$ converges monotonically to a real number $\beta_{0}$ as $k \rightarrow \infty$. Using (2.14a), one also has

$$
\frac{\lambda}{2} \sum_{k \geqslant 0}\left|p_{k}-p_{k+1}\right|_{H}^{2} \leqslant J^{*}\left(p_{0}\right)-\beta_{0},
$$

whence

$$
\lim _{k \rightarrow \infty}\left|p_{k}-p_{k+1}\right|_{H}=0
$$

(obviously, it may be assumed that $p_{0} \in \operatorname{Dom} g^{*}$ ). Since $J\left(u_{k}\right)$ is also uniformly bounded, (2.9) yields

$$
u_{k} \text { is uniformly bounded in } V \text {. }
$$

This proves that $\left\{u_{k}\right\}$ possesses weak adherent points in $V$.

Suppose now that $u$ is the weak limit of a subsequence $\left\{u_{\mu}\right\}$. We know that

$$
p_{\mu} \in \partial g\left(B u_{\mu}+\lambda\left(p_{\mu-1}-p_{\mu}\right)\right) \text { for all } \mu \text {. }
$$

But $B u_{\mu}+\lambda\left(p_{\mu-1}-p_{\mu}\right)$ converges strongly in $H$ towards $B u$. Hence, $\left\{p_{\mu}\right\}$ is bounded and it can be assumed that it converges weakly in $H$ to $p$. To conclude, it will be sufficient to prove that

$$
B^{*} p \in \partial f(u) \cap B^{*} \partial g(B u) .
$$

Now, from (2.17) one sees that $p$ is also the weak limit of $p_{\mu-1}$ as $\mu \rightarrow \infty$. Therefore, the lower semicontinuity of $f$ and $g$ gives

$$
\begin{aligned}
& f(u)+f^{*}\left(B^{*} p\right) \leqslant \frac{\lim }{\mu \rightarrow \infty}\left\{f\left(u_{\mu}\right)+f^{*}\left(B^{*} p_{\mu-1}\right)\right\} \\
& =\lim _{\mu \rightarrow \infty}\left(p_{\mu \cdot 1}, B u_{\mu}\right)_{\|}=(p, B u)_{\|}, \\
& g(B u)+g^{*}(p) \leqslant \frac{\lim }{\mu \rightarrow \infty}\left\{g\left(B u_{\mu}+\lambda\left(p_{\mu-1}-p_{\mu}\right)\right)+g^{*}\left(p_{\mu}\right)\right\} \\
& =(p, B u)_{\|},
\end{aligned}
$$

and the proof is completed. 
The function $J^{*}$, given by

$$
J^{*}(q)=g^{*}(q)-f^{*}\left(B^{*} q\right) \quad \forall q \in H,
$$

is the dual (or polar) functional associated with $J$ (cf. [41], [42]).

Next, we make some remarks regarding Algorithm 1. Hypotheses (2.8) and/or (2.9) can be replaced, respectively, by

$$
\begin{gathered}
\inf _{q \in H} J^{*}(q) \geqslant-C>-\infty, \\
J^{*} \text { is coercive. }
\end{gathered}
$$

We also note that

$$
p \in \partial g(B u), \quad B^{*} p \in \partial f(u) \leftrightarrow u \in \partial f^{*}\left(B^{*} p\right), \quad B u \in \partial g^{*}(p) .
$$

In other words, finding critical points for $J$ and $J^{*}$ are, in some sense, equivalent problems. It is thus reasonable to formulate iterative schemes of the kind (2.4) for the dual problem

$$
\partial g^{*}(p)-B \partial f^{*}\left(B^{*} p\right) \ni 0, \quad p \in H .
$$

The previous algorithm is related to Algorithm 2 in [17]. There, $u_{k+1}$ and $p_{k+1}$ are defined recursively, using a semi-implicit gradient formula for the (totally) regularized problem

$$
\begin{aligned}
& \text { Minimize } f_{\lambda}(v)-g_{\lambda}(v) \\
& \text { subject to } v \in V .
\end{aligned}
$$

Other than that, we have adopted a different point of view, carrying out a partial, or at least independent, exact regularization procedure for the function $J$. Some advantages of this are that similar arguments hold for certain constrained problems (cf. Section 4) and that local features, typical of certain operators arising in differential problems, are, in this way, preserved.

3. Applications (I): Some Elliptic Problems with Discontinuous Nonlinearities. As a first application, consider the following problem:

$$
\begin{aligned}
& \text { Find } u \in H^{2}(\Omega) \text { such that }-\Delta u(x) \in \partial G(x, u(x)) \text { a.e. in } \Omega, \\
& u=0 \text { on } \partial \Omega \text {. }
\end{aligned}
$$

Here, $\Omega \subset \mathbf{R}^{N}$ is a bounded open set whose boundary $\partial \Omega$ is sufficiently smooth and $G: \bar{\Omega} \times \mathbf{R} \rightarrow \mathbf{R}$ is continuous, convex and subquadratic with respect to its second variable, i.e.,

$$
G(x, s) \leqslant \frac{a}{2}|s|^{2}+b \quad \forall(x, s) \in \bar{\Omega} \times \mathbf{R}, a<\lambda_{1} .
$$

$\lambda_{1}$ is the first eigenvalue of the homogeneous Dirichlet problem for the operator $-\Delta$; in the sequel, $\partial G(x, \hat{s})$ stands for the subdifferential of the function $s \rightarrow G(x, s)$ at $\hat{s}$. 
It is not difficult to see that (3.1) can be rewritten in the form (2.3) with

$$
\begin{aligned}
& V=H_{0}^{1}(\Omega), \quad H=L^{2}(\Omega), \quad B: \text { the compact embedding } H_{0}^{1} \hookrightarrow L^{2}, \\
& f(v) \equiv \frac{1}{2} \int_{\Omega}|\nabla v|^{2} d x \quad \text { and } \quad g(q) \equiv \int_{\Omega} G(x, q) d x .
\end{aligned}
$$

In this case, Algorithm 1 leads to the following iteration:

$$
\begin{aligned}
& -\Delta u_{k+1}=p_{k} \quad \text { in } \Omega, \quad u_{k+1}=0 \text { on } \partial \Omega, \\
& p_{k+1}(x)=(\partial G)_{\lambda}\left(x, u_{k+1}(x)+p_{k}(x)\right) \quad \text { a.e. in } \Omega .
\end{aligned}
$$

Since $G$ is subquadratic in $s$, assumptions (2.8) and (2.9) are satisfied. Hence, from the convergence result in Section 2 and standard elliptic regularity one easily deduces the strong convergence in $H_{0}^{1}(\Omega)$ of a subsequence $\left\{u_{k}\right\}$ towards a solution of (3.1).

From a computational point of view, the preceding method is not expensive; at each step, it only requires the solution of a linear problem and the computation of $p_{k+1}$. This can be easily achieved if we perform previously a tabulation of the function $(\partial G)_{\lambda}$ and approximate to $p_{k+1}(x)$ using an interpolation method.

Roughly speaking, (3.3) plays the role of the monotone scheme of H. Amann [1], [2] and D. Sattinger [35] in the case in which $G(x, \cdot)$ is not differentiable. Let us point out that the sequence $\left\{u_{k}\right\}$ is monotonic if one chooses $p_{0}$ appropriately (for a precise result, see [12]). A negative aspect of (3.3) is, however, that the positiveness of $\lambda$ may decrease the speed of convergence. Indeed, it is straightforward to verify that, when $\left\{u_{k}\right\}$ is monotonic, the larger $\lambda$ is, the more slowly $u_{k}$ converges. It is thus quite tempting to conclude that a more appropriate algorithm would be obtained if $\lambda$ were replaced by $-\lambda$ in (3.3), i.e., by setting

$$
p_{k+1} \in-\frac{1}{\lambda}\left(\operatorname{Id}-(\operatorname{Id}-\lambda \partial G(x, \cdot))^{-1}\right)\left(u_{k+1}-\lambda p_{k}\right) \text { a.e. }
$$

In fact, using (3.2), it can be proved that, for small $\lambda>0$, the operator

$$
-\frac{1}{\lambda}\left(\operatorname{Id}-(\operatorname{Id}-\lambda \partial G(x, \cdot))^{-1}\right): \mathbf{R} \rightarrow \mathbf{R}
$$

is single-valued for $x$ a.e. in $\Omega$.

More generally, with the setting of Section 2, one has the following result, which can be demonstrated similarly to Theorem 2.7 .

THEOREM 3.6. Assume that, together with (2.6), (2.8)-(2.10), one has

$$
\exists a, b>0 \text { such that } g(q) \leqslant \frac{a}{2}|q|_{H}^{2}+b \quad \forall q \in H .
$$

Then there exists $\tilde{\lambda}>0$, only depending on $a$, fand $|B|_{\mathscr{L}(V ; H)}$, such that:

(i) For any $\lambda \in(0, \tilde{\lambda})$, the operator $(\partial g)_{-\lambda}=-\left(\mathrm{Id}-(\mathrm{Id}-\lambda \partial g)^{-1}\right) / \lambda$ is singlevalued, with domain $H$;

(ii) For any $p_{0} \in H$ and any $\lambda \in(0, \tilde{\lambda})$, the iterates in

$$
\partial f\left(u_{k+1}\right) \ni B p_{k}, \quad p_{k+1}=(\partial g)_{-\lambda}\left(B u_{k+1}-\lambda p_{k}\right)
$$

are well defined. The conclusion of Theorem 2.7 holds for the sequence $\left\{u_{k}\right\}$.

4. Some Problems with Constraints on the Boundary of a Convex Set. With the general setting of Section 2, let $K$ be a closed convex subset of $H$ whose boundary $\partial K$ is nonempty. We wish to solve the following extremal problem, formulated in 
terms of the (originally) dual functional:

$$
\begin{aligned}
& \text { Minimize } g^{*}(q)-f^{*}\left(B^{*} q\right) \\
& \text { subject to } q \in \partial K
\end{aligned}
$$

As mentioned above (see Section 1), the following optimality condition must hold at a solution $p \in \partial K$ :

$$
\partial g^{*}(p)-\partial\left(f^{*} \circ B^{*}\right)(p)+N(K ; p) \cap N\left(K^{c} ; p\right) \ni 0 .
$$

Set $N_{\partial K}(p)=N(K ; p) N\left(K^{c} ; p\right) ; N_{\partial K}(p)$ is the normal subcone to $\partial K$ at $p$. In order to find a solution to (4.2), we propose the following "formal" scheme, which in spirit agrees with that of Algorithm 1.

Algorithm 2 ( $\lambda$ is a fixed positive parameter).

(a) Choose $p_{0} \in H$.

(b) For given $k \geqslant 0$ and $p_{k} \in H$, compute $v_{k+1}$ and $p_{k+1}$ by

$$
\begin{aligned}
& v_{k+1} \in \partial f^{*}\left(B^{*} p_{k}\right)+B^{-1} N_{\partial K}\left(p_{k}\right), \\
& p_{k+1}=g_{\lambda}^{\prime}\left(B v_{k+1}+\lambda p_{k}\right), \quad p_{k+1} \in \partial K .
\end{aligned}
$$

These iterates must be interpreted as follows: The choice of the component of $v_{k+1}$ in $B^{-1} N_{\partial K}\left(p_{k}\right)$ has to be such that, for $p_{k+1}$ as in (4.3), one has $p_{k+1} \in \partial K$.

In order to make (4.3) meaningful, it will be assumed that (2.6) still holds and that

$$
R(B) \cap N_{\partial K}(q) \neq \varnothing \quad \forall q \in \partial K .
$$

The regularity property (1.17) is satisfied by a large number of constraint sets. When it holds, $N_{\partial K}(q)$ is a straight line for every $q \in \partial K$, spanned by the (normal) vector $\eta(q) \neq 0$. It is obvious that, in this case, (4.3) can be restated as:

$$
\begin{aligned}
& \text { Find } w_{k+1} \in \partial f^{*}\left(B^{*} p_{k}\right) \text { and } \beta_{k+1} \in \mathbf{R} \text { such that } \\
& g_{\lambda}^{\prime}\left(B w_{k+1}+\beta_{k+1} \cdot \eta\left(p_{k}\right)+\lambda p_{k}\right) \in \partial K
\end{aligned}
$$

In other words, under assumptions (1.17) and (4.4), (4.3) differs from (2.4) exclusively in the solution of a nonlinear scalar problem in the real variable $\beta_{k+1}$. In the remaining parts of this paper, we illustrate the behavior of Algorithm 2 with two applications.

5. Applications (II): A Free Boundary Problem in Plasma Physics. Let $\Omega$ be a bounded open set in $\mathbf{R}^{2}$ with smooth boundary $\partial \Omega$. We consider the following semilinear elliptic problem, which models the equilibrium of a plasma confined in a cylindrical cavity:

$$
\text { Find } u \in H^{2}(\Omega) \text { and } \beta \in \mathbf{R} \text { such that }-\Delta u(x) \in \partial G(x, u(x))
$$

$$
\text { a.e. in } \Omega, u=\beta \text { on } \partial \Omega,-\int_{\partial \Omega} \frac{\partial u}{\partial n} d \Gamma=I \text {. }
$$


Here, $I$ is a positive constant (the total longitudinal current interior to the plasma), $u=u\left(x_{1}, x_{2}\right)$ is the flux function for the magnetic field and $G: \bar{\Omega} \times \mathbf{R} \rightarrow \mathbf{R}$ is required to satisfy:

$G$ is continuous, $s \rightarrow G(x, s)$ is convex $\forall x \in \bar{\Omega}$, $G(x, s)=0$ for $s \leqslant 0$, $\exists c_{1}, c_{2} \in \mathbf{R}$ such that $G(x, s) \leqslant c_{1} s^{2} / 2+c_{2}$,

$$
\lim _{s \rightarrow \infty} \int_{\Omega} G(x, s-0) d x>I \text {. }
$$

In view of (G.1)-(G.2), $\partial G$ is nondecreasing in $s$, and one can write

$$
\partial G(x, s)=[\partial G(x, s-0), \partial G(x, s+0)] \quad \forall(x, s) \in \bar{\Omega} \times \mathbf{R} .
$$

If $(u, \beta)$ is a solution of $(5.1)$ and $\beta<0$, a free boundary is implicitly given by (5.1): the boundary of the open set

$$
\Omega_{p}=\{x \in \Omega ; u(x)>0\},
$$

i.e., the cross section of the cylindrical region occupied by the plasma. For a complete derivation of the equations which lead to (5.1) see [27], [39]. Problem (5.1) and/or some of its variants have been studied by several authors (see, e.g., [7], [24], [30], [36], [39], [40]). From a numerical point of view, an iterative procedure has been analyzed by $\mathrm{H}$. Berestycki and $\mathrm{H}$. Brézis in [7] (see also [37]).

In the context of Section 4, (5.1) possesses a weak formulation with

$$
\begin{aligned}
& V=H_{0}^{1}(\Omega) \oplus \mathbf{R}, \quad H=L^{2}(\Omega), \quad K=\left\{q \in H: \int_{\Omega} q d x \leqslant I\right\}, \\
& B: \text { the compact embedding } H_{0}^{1} \oplus \mathbf{R} \hookrightarrow L^{2} \\
& f(v) \equiv \frac{1}{2} \int_{\Omega}|\nabla v|^{2} d x \text { and } g(q) \equiv \int_{\Omega} G(x, q) d x
\end{aligned}
$$

In this case, the set $K$ is a closed semispace of $L^{2}(\Omega)$ and

$$
\partial K=\left\{q \in L^{2}(\Omega) ; \int_{\Omega} q d x=I\right\}
$$

consequently, $N_{\partial K}(q) \equiv \mathbf{R}$ for every $q \in K$ and assumptions (1.17) and (4.4) are satisfied. Also, it is not difficult to prove that (2.6) holds.

Assuming that Algorithm 2 is well defined, i.e., (4.3) possesses a solution for each $k \geqslant 0$, it will lead to the following iteration:

$$
\begin{aligned}
& -\Delta w_{k+1}=p_{k} \quad \text { in } \Omega, \quad w_{k+1}=0 \quad \text { on } \partial \Omega \\
& p_{k+1}=g^{\prime}\left(B w_{k+1}+\beta_{k+1}+\lambda p_{k}\right), \quad \int_{\Omega} p_{k+1} d x=I, \quad \beta_{k+1} \in \mathbf{R} .
\end{aligned}
$$

Thus, we have to prove that, for a given $p_{k} \in \partial K$ satisfying $p_{k} \geqslant 0$ a.e., there exists a solution $\beta_{k+1}$ to (5.3). We begin by stating the following lemma, whose proof is given below. 
LEMMA 5.4. Under assumptions (G.1)-(G.4) one has

$$
\lim _{\beta \uparrow \infty} \int_{\Omega} g^{\prime}(\beta) d x>I
$$

For any $\beta \in \mathbf{R}$, set

$$
T_{k}(\beta)=\beta-\frac{\lambda}{\operatorname{meas}(\Omega)}\left\{\int_{\Omega} g^{\prime}\left(B w_{k+1}+\beta+\lambda p_{k}\right) d x-I\right\} .
$$

Evidently, $\beta$ is a solution of (5.3) if and only if it is a fixed point of $T_{k}$. The function $\beta \rightarrow T_{k}(\beta)$ is nondecreasing; thus, if we choose $\beta_{0, k}$ arbitrarily in $\mathbf{R}$ and set

$$
\beta_{l+1, k}=T_{k}\left(\beta_{l, k}\right) \quad \forall l \geqslant 0,
$$

the sequence $\left\{\beta_{l, k}\right\}_{l}$ is bounded independently of $k$ and $\beta_{0, k}$. Indeed, let us first assume that it is nondecreasing and let $\beta_{*}$ be such that

$$
\int_{\Omega} g^{\prime}\left(\beta_{*}\right) d x>I
$$

Then $\beta_{l, k}<\beta_{*}$ for all $l \geqslant 0$; otherwise if $\beta_{l, k} \geqslant \beta_{*}$ for a certain $l$, one would have

$$
\begin{aligned}
\beta_{l, k}-\beta_{l+1, k} & =\frac{1}{\operatorname{meas}(\Omega)}\left\{\int_{\Omega} g^{\prime}\left(\beta_{l, k}\right) d x-I\right\} \\
& \geqslant \frac{1}{\operatorname{meas}(\Omega)}\left\{\int_{\Omega} g^{\prime}\left(\beta_{*}\right) d x-I\right\}
\end{aligned}
$$

and $\beta_{l, k}>\beta_{l+1, k}$, which contradicts the fact that $\left\{\beta_{l, k}\right\}_{l}$ is nondecreasing.

On the other hand, if $\left\{\beta_{l, k}\right\}_{l}$ is nonincreasing, it must be bounded from below by a constant $\beta_{* *}$ independent of $k$ and $\beta_{0, k}$. Indeed, from elliptic regularity (see, e.g., [20]), one knows that a positive constant $\delta$ exists (only depending on $I$ and $\Omega$ ) such that

$$
\left|w_{k+1}\right|_{L^{1}}=\int_{\Omega} w_{k+1} d x \geqslant \delta .
$$

Clearly, $\lim _{\beta \downarrow-\infty} \int_{\Omega} g_{\lambda}^{\prime}(\beta) d x=0$. Let $\beta_{* *}$ be such that

$$
\int_{\Omega} g^{\prime}\left(\beta_{* *}\right) d x<\delta / 2
$$

Then $\beta_{l, k}>\beta_{* *}$ for all $l \geqslant 0$, for, if $\beta_{l, k} \leqslant \beta_{* *}$ for a certain $l$, one would have

$$
\begin{aligned}
\beta_{l+1, k}-\beta_{l, k} & =\frac{1}{\operatorname{meas}(\Omega)}\left\{\delta-\int_{\Omega} g^{\prime}\left(\beta_{l, k}\right) d x\right\} \\
& \geqslant \frac{\delta}{2 \cdot \operatorname{meas}(\Omega)},
\end{aligned}
$$

whence $\beta_{l+1, k}>\beta_{l, k}$, which is absurd.

Therefore, the sequence $\left\{\beta_{l, k}\right\}$, converges, and its limit $\beta_{* k}$ is a solution of (5.3). Since the bounds $\beta_{*}$ and $\beta_{* *}$ only depend on $\lambda, g, I$ and $\Omega$, we conclude that (5.3) can be solved for each $k$ with solutions $\beta_{k+1}=\beta_{* k}$ in a bounded interval of $\mathbf{R}$. 
It remains to prove Lemma 5.4 .

Proof of Lemma 5.4. It will suffice to prove that, for $\beta$ large enough,

$$
\int_{\Omega}\left\{\beta-R^{g}(\beta)\right\} d x-\lambda I>0 .
$$

By the definition of $R_{\lambda}^{g}$, one must have

$$
\beta \in R_{\lambda}^{g}(\beta)+\lambda\left[\partial G\left(x, R_{\lambda}^{g}(\beta)-0\right), \partial G\left(x, R_{\lambda}^{g}(\beta)+0\right)\right] \quad \text { a.e., }
$$

whence $\beta \geqslant R_{\lambda}^{g}(\beta)+\lambda \partial G\left(x, R_{\lambda}^{g}(\beta)-0\right)$, and it suffices to prove

$$
\int_{\Omega} \partial G\left(x, R_{\lambda}^{\xi}(\beta)-0\right) d x>I \text { for some } \beta .
$$

But this is an almost trivial consequence of (G.4).

Once it has been established that Algorithm 2 is well defined for problem (5.1), a convergence result for (5.3) can be derived:

THEOREM 5.11. Assume that $G$ satisfies (G.1)-(G.4), and let $p_{0}$ be a function in $\partial K$ a.e. nonnegative. Then the sequence $\left(w_{k}, \beta_{k}\right)$, given by (5.3), possesses subsequences which converge strongly in $H_{0}^{1}(\Omega) \times \mathbf{R}$. If $(w, \beta)$ is the limit of such a sequence, then the pair $(w+\beta, \beta)$ is a solution of (5.1).

Proof. Using the same argument as in the proof of Theorem 2.7, one obtains

$$
\begin{aligned}
J^{*}\left(p_{k+1}\right) & \leqslant f\left(w_{k+1}\right)+g^{*}\left(p_{k+1}\right)-\int_{\Omega} p_{k+1} w_{k+1} d x \\
& \leqslant J^{*}\left(p_{k}\right)-\frac{\lambda}{2} \int_{\Omega}\left|p_{k}-p_{k+1}\right|^{2} d x \quad \forall k \geqslant 0 .
\end{aligned}
$$

Furthermore, $J^{*}$ is coercive and bounded from below in $\partial K$. Indeed, for any $q \in \partial K$, one has (here $c$ and $\hat{c}$ are positive constants and $S$ is the inverse of $-\Delta$ with homogeneous Dirichlet conditions):

$$
\begin{aligned}
J^{*}(q) & =\int_{\Omega} G^{*}(x, q) d x-\frac{1}{2} \int_{\Omega} S B^{*} q \cdot q d x \\
& \geqslant \frac{1}{2 c}|q|_{L^{2}}^{2}-\frac{I}{2} c|q|_{L^{2}}-\hat{c} .
\end{aligned}
$$

As in the proof of Theorem 2.7 , one deduces that $\left(p_{k}, \beta_{k}\right)$ is uniformly bounded in $L^{2}(\Omega) \times \mathbf{R}$. So, $w_{k}$ is uniformly bounded in $H^{2}(\Omega)$ and the existence of convergent subsequences is demonstrated.

Let $(w, \beta)$ be the strong limit in $H_{0}^{1}(\Omega) \times \mathbf{R}$ of a subsequence $\left\{\left(w_{\mu}, \beta_{\mu}\right)\right\}$. We may suppose that $p_{\mu} \rightarrow p$ weakly in $L^{2}(\Omega)$, with $p \in \partial K$. Also, $p_{\mu}-p_{\mu-1} \rightarrow 0$ strongly; from relations

$$
p \in \partial g\left(B w_{\mu}+\beta_{\mu}+\lambda\left(p_{\mu}-p_{\mu-1}\right)\right), \quad w_{\mu}=S B^{*} p_{\mu-1},
$$

it is easily seen that $p \in \partial g(B w+\beta)$ and $w=S B^{*} p$, i.e.,

$$
\begin{aligned}
& p(x) \in \partial G(x, w(x)+\beta) \quad \text { a.e. in } \Omega, \\
& -\Delta w=p \quad \text { in } \Omega, w \in H_{0}^{1}(\Omega) .
\end{aligned}
$$

As a consequence, $(w+\beta, \beta)$ solves $(5.1)$. 
As in the case of problem (3.1), one observes that, for $\lambda=0$, at least formally, (5.3) coincides with the fixed-point method described in [7]. Certain uniqueness results may be derived for (5.1) under some additional assumptions on $G$ (cf. [7], [40]). Obviously, if the solution of (5.1) is unique, then the whole sequence $\left\{\left(w_{k}, \beta_{k}\right)\right\}$ converges.

6. Applications (III): A Free Boundary Problem in Vortex Ring Theory. We finally present an application of the previous techniques to another free boundary problem, this time originating in fluid mechanics. For a given bounded open set $\Omega \subset \mathbf{R}^{2}$ with smooth boundary $\partial \Omega$, we wish to

Find $u \in H^{2}(\Omega)$ and $W>0$ such that $-\Delta u(x) \in H(u(x)-W \zeta(x))$

$$
\text { a.e. in } \Omega, u=0 \text { on } \partial \Omega, \int_{\Omega}|\nabla u|^{2} d x=\eta \text {. }
$$

Here, $\eta$ is a prescribed positive constant,

$$
\zeta \in C^{1}(\bar{\Omega}), \quad \zeta>0 \text { in } \Omega,
$$

and $H$ is, up to a positive coefficient $\alpha$, the (maximal monotone) operator associated with Heaviside's function, i.e.,

$$
H(s)= \begin{cases}0 & \text { for } s \leqslant 0 \\ {[0, \alpha]} & \text { for } s=0(\alpha>0), \\ \alpha & \text { for } s>0\end{cases}
$$

Problem (6.1) serves to model the equilibrium of a (plane) vortex pair in an ideal fluid with vanishing flux parameter (cf. [29]; for a similar problem corresponding to the equilibrium of an axisymmetric vortex ring, see [15]). It is easy to see that (6.1) is in fact a free boundary problem, where the unknown subdomain is the so-called vorticity core region

$$
\Omega_{v}=\{x \in \Omega ; u(x)-W \zeta(x)>0\} .
$$

Semilinear elliptic problems of this kind have been studied by many authors (see [3], [5], [6], [8], [16], [28]). In (6.1), the original domain (unbounded) has been approximated by $\Omega$, as in [6], [8].

In the sequel, we will assume that the following assumption is satisfied:

Assumption (A). Let $u \in C^{2}(\bar{\Omega})$ be the solution of

$$
-\Delta u=\alpha \quad \text { in } \Omega, \quad u=0 \quad \text { on } \partial \Omega .
$$

Then

$$
\int_{\Omega} u_{\alpha} d x=\int_{\Omega}\left|\nabla u_{\alpha}\right|^{2} d x>\eta
$$

It is straightforward to see that (A) is in fact a necessary condition for the existence of a solution of (6.1).

Clearly, (6.1) can be rewritten as a problem of type (4.1). It suffices to set

$$
V=H^{1}(\Omega), \quad H=L^{2}(\Omega), \quad K=\left\{q \in H ; \int_{\Omega} S B^{*} q \cdot q d x \leqslant \eta\right\},
$$


with $B$ the compact embedding $H^{1} \hookrightarrow L^{2}, S$ as in Section 5,

$$
f(v) \equiv \begin{cases}0 & \text { if } v+\zeta=0, \quad g(q) \equiv \alpha \int_{\Omega} q_{+} d x, \quad q_{+}=\max (q, 0) . \\ +\infty & \text { otherwise, }\end{cases}
$$

Then $K$ is a closed convex subset of $L^{2}(\Omega)$ whose boundary is given by

$$
\partial K=\left\{q \in L^{2}(\Omega) ; \int_{\Omega} S B^{*} q \cdot q d x=\eta\right\} .
$$

It is not hard to check that (1.19) and (4.4) hold, since

$$
N_{\partial K}(q)=\left\{\kappa B S B^{*} q ; \kappa \in \mathbf{R}\right\} \quad \forall q \in \partial K .
$$

Also, (2.6) is fulfilled.

Note that Algorithm 2 leads to the following iteration:

$$
-\Delta w_{k+1}=p_{k} \quad \text { in } \Omega, \quad w_{k+1}=0 \quad \text { on } \partial \Omega,
$$

$$
\begin{aligned}
& p_{k+1}=g_{\lambda}^{\prime}\left(B\left(\nu_{k+1} w_{k+1}-\zeta\right)+\lambda p_{k}\right), \quad \int_{\Omega} S B^{*} p_{k+1} \cdot p_{k+1} d x=\eta, \\
& \nu_{k+1} \in \mathbf{R} .
\end{aligned}
$$

Now, for given $q \in L^{2}(\Omega)$, one has

$$
\hat{q}=g_{\lambda}^{\prime}(q) \leftrightarrow \hat{q}(x)= \begin{cases}0 & \text { if } q(x)<0, \\ q(x) / \lambda & \text { if } 0 \leqslant q(x) \leqslant \lambda \alpha, \\ \alpha & \text { if } q(x)>\lambda \alpha .\end{cases}
$$

For any $\nu \in \mathbf{R}$, set

$$
E_{k}(\nu)=\int_{\Omega} S B^{*} p_{\nu} \cdot p_{\nu} d x \quad \text { with } p_{\nu}=g_{\lambda}^{\prime}\left(B\left(\nu w_{k+1}-\zeta\right)+\lambda p_{k}\right) .
$$

Then $\nu_{k+1}$ solves (6.5) if and only if it solves the scalar equation

$$
E_{k}(\nu)=\eta \text {. }
$$

Arguing as in Section 5, it can be proved that there exist positive constants $\lambda_{*}$ and $\beta$ (only depending on $\alpha, \eta$ and $\Omega$ ) such that, if $0<\lambda<\lambda_{*}$, (6.6) possesses a solution $\nu_{k+1} \geqslant \beta$. Set

$$
D_{k}(\nu)=\nu-\frac{\lambda}{\operatorname{meas}(\Omega)}\left\{E_{k}(\nu)-\eta\right\}
$$

then a rather natural procedure for solving (6.6) is given by

$$
\nu_{l+1}^{k+1}=D_{k}\left(\nu_{l}^{k+1}\right), \quad l \geqslant 0 .
$$

We end this section with a convergence result for (6.5), which can be proved similarly to Theorem 5.11 .

THEOREM 6.7. Assume that Assumption (A) is satisfied and take $\lambda$ in $\left(0, \lambda_{*}\right)$, $p_{0} \in \partial K, 0 \leqslant p_{0} \leqslant \alpha$ a.e. Then the sequence $\left\{\left(u_{k}, 1 / \nu_{k}\right)\right\}$, defined by (6.5), possesses subsequences which converge strongly in $H_{0}^{1}(\Omega) \times \mathbf{R}_{+}$. If $(u, W)$ is the limit of such a subsequence, then it is a solution of (6.1). 
Again, (6.5) is a formal generalization of the fixed-point method used by $\mathrm{H}$. Berestycki et al. in [8]. The main advantages of this procedure are that it permits the numerical treatment of the discontinuous nonlinearity and also that a convergence result has been derived.

Acknowledgment. The authors are indebted to Professor J. Hernández (Universidad Autónoma, Madrid) for several fruitful discussions regarding problem (3.1).

Facultad de Matemáticas

Universidad de Sevilla

C/Tarfia s/n

41012 Sevilla, Spain

Facultad de Ciencias

Universidad Autónoma

28034 Madrid, Spain

1. H. AmanN, "Existence and stability of solutions for semilinear parabolic systems and applications to some diffusion-reaction equations," Proc. Roy. Soc. Edinburgh Sect. A, v. 81, 1978, pp. 37-47.

2. H. AmanN, "On the existence of positive solutions of nonlinear elliptic boundary value problems," Indiana Univ. Math. J., v. 21, 1971, pp. 125-146.

3. A. Ambrosetri \& G. Mancini, "Remarks on some free boundary problems," in Recent Contributions to Nonlinear Partial Differential Equations (H. Berestycki and H. Brézis, eds.), Pitman, London, 1981.

4. G. Auchmuty, “Duality for nonconvex variational principles,” J. Differential Equations, v. 50, 1983, pp. $80-145$.

5. T. B. Benjamin, The Alliance of Practical and Analytical Insights into the Nonlinear Problems of Fluid Mechanics, Lecture Notes in Math., vol. 503, Springer-Verlag, Berlin and New York, 1976, pp. 8-29.

6. H. BERESTYCKI, Thesis, University of Paris VI, 1980.

7. H. BERESTYCKI \& H. BREZIS, "On a free boundary problem arising in plasma physics," Nonlinear Anal. T.M.A., v. 4, 1980, pp. 415-436.

8. H. Berestycki, E. Fernández-CARA \& R. Glowinski, "A numerical study of some questions in vortex ring theory," RAIRO Anal. Numér., v. 18, 1984, pp. 7-85.

9. A. BermUdez \& C. Moreno, "Duality methods for solving variational inequalities," Comput. Math. Appl., v. 7, 1981, pp. 43-58.

10. F. H. Clarke, “Generalized gradients and applications,” Trans. Amer. Math. Soc., v. 205, 1975, pp. 247-262.

11. W. FenChEL, “On conjugate convex functions,” Canad. J. Math., v. 1, 1949, pp. 73-77.

12. E. FernÁNDEZ-CARA \& C. MoReno, "Exact regularization and critical point approximation," in Contributions to Nonlinear Partial Differential Equations, Vol. II (I. Díaz and P. L. Lions, eds.). (To appear.)

13. M. FORTIN, "Minimization of some nondifferentiable functionals by the augmented Lagrangian method of Hestenes and Powell," Appl. Math. Optim., v. 2, 1976, pp. 236-250.

14. M. Fortin \& R. Glowinski, Augmented Lagrangian Methods: Applications to the Numerical Solution of Boundary-Value Problems, North-Holland, Amsterdam, 1983.

15. L. E. Fraenkel \& M. S. Berger, "On the global theory of vortex rings in an ideal fluid," Acta Math., v. 132, 1974, pp. 13-51.

16. A. Friedman \& B. Turckington, “Asymptotic estimates for an axisymmetric rotating fluid," $J$. Funct. Anal., v. 37, 1980, pp. 136-163.

17. D. Gabay, "Minimizing the difference of two convex functions: Algorithms based on exact regularization." (To appear.)

18. D. GaBay, Chapter 9 in Reference 14.

19. D. GabAy \& B. MerCIER, "A dual algorithm for the solution of nonlinear variational problems via finite element approximations," Comput. Math. Appl., v. 2, 1976, pp. 17-40.

20. D. Gilbarg \& N. S. Trudinger, Elliptic Partial Differential Equations of the Second Order, Springer-Verlag, Berlin and New York, 1977.

21. R. Glowinski, Numerical Methods for Nonlinear Variational Problems, 2nd ed., Springer-Verlag, Berlin and New York, 1984. 
22. R. Glowinski \& A. Marrocco, "Sur l'approximation par éléments finis d'ordre un et la résolution par pénalisation-dualité d'une classe de problèmes de Dirichlet non linéaires," RAIRO Anal. Numér. Ser. Rouge, v. 2, 1975, pp. 41-76.

23. J. B. HiriaRT-URRUTY, "Tangent cones, generalized gradients and mathematical programming in Banach spaces," Math. Oper. Res., v. 4, 1979, pp. 79-97.

24. D. Kinderlehrer, L. Nirenberg \& J. SpruCK, "Regularity in elliptic free boundary problems. II," J. Analyse Math., v. 34, 1978, pp. 86-119.

25. P. L. Lions \& B. MerCier, "Splitting algorithms for the sum of two nonlinear operators," SIAM J. Numer. Anal., v. 16, 1979, pp. 964-979.

26. B. Martinet, “Détermination approchée d'un point fixe d'une application pseudo-contractante.

Cas de l'application proximale," C. R. Acad. Sci. Paris Sér. A, v. 274, 1972, pp. 163-165.

27. C. MERCIER, The Magnetohydrodynamic Approach to the Problem of Plasma Confinement in Closed Magnetic Configurations, EURATOM-CEA, Comm. of the European Communities, Luxembourg, 1974 (Rep. EUR 5127/1).

28. Wei-Ming Ni, "On the existence of global vortex rings," J. Analyse Math., v. 37, 1980, pp. $208-247$.

29. J. Norbury, "Steady planar vortex pairs in an ideal fluid," Comm. Pure Appl. Math., v. 28, 1975, pp. $679-700$.

30. J. M. Puel, "Sur un problème de valeur propre nonlinéaire et de frontiere libre," C. R. Acad. Sci. Paris Ser. A, v. 284, 1977, pp. 861-863.

31. R. T. Rockafellar, "Monotone operators and the proximal point algorithm," SIAM J. Control Optim., v. 14, 1976, pp. 877-898.

32. R. T. Rockafellar, "Augmented Lagrangians and applications of the proximal point algorithm in convex programming," Math. Oper. Res., v. 1, 1976, pp. 97-116.

33. R. T. Rockafellar, "The multipliers method of Hestenes and Powell applied to convex programming," J. Optim. Theory Appl., v. 12, 1973, pp. 555-562.

34. R. T. Rockafellar, Convex Functions and Duality in Optimization Problems and Dynamics, Lecture Notes Op. Res. Math. Ec., Vol. II, Springer-Verlag, Berlin, 1969.

35. D. Sattinger, "Monotone methods in nonlinear elliptic and parabolic equations," Indiana Univ. Math. J., v. 21, 1972, pp. 979-1000.

36. D. F. SchaefFer, "Nonuniqueness in the equilibrium shape of a confined plasma," Comm. Partial Differential Equations, v. 2, 1977, pp. 587-600.

37. M. Sermange, Thesis, University of Paris XI, 1982.

38. I. Stakgold, "Estimates for some free boundary problems," in Ordinary and Partial Differential Equations Proc., Dundee 1980 (W. N. Everitt and B. D. Sleeman, eds.), Lecture Notes in Math., vol. 846, Springer-Verlag, Berlin, 1981.

39. R. Tемам, "A nonlinear eigenvalue problem: The shape at equilibrium of a confined plasma," Arch. Rational Mech. Anal., v. 60, 1975, pp. 51-73.

40. R. TEмам, "Remarks on a free boundary problem arising in plasma physics," Comm. Partial Differential Equations, v. 2, 1977, pp. 563-585.

41. J. F. Toland, "A duality principle for nonconvex optimization and the calculus of variations," Arch. Rational Mech. Anal., v. 71, 1979, pp. 41-61.

42. J. F. Toland, “Duality in nonconvex optimization," J. Math. Anal. Appl., v. 66, 1978, pp. 399-415. 